\title{
Photonics in photovoltaic systems
}

\author{
Andreas Gombert ${ }^{*}, 1$ and Antonio Luque ${ }^{2}$ \\ Concentrix Solar GmbH, Bötzinger Str. 31, 79111 Freiburg, Germany \\ Universidad Politécnica de Madrid, Instituto de Energía Solar, 28040 Madrid, Spain
}

\begin{abstract}
This paper gives an overview on photonics for photovoltaic systems. Starting from the spectral and angular distribution of the electromagnetic radiation from the sun, many important optical approaches how to improve the efficiency of solar cells are presented and discussed. Topics include antireflec-
\end{abstract}

tive coatings, various light trapping structures, refractive, reflective and fluorescent concentrators, and components for spectral management. The theoretical background is shortly described and examples of the experimental and also of the commercial realisation are given.
1 Introduction The conversion of solar energy to electrical energy by using photovoltaic (PV) cells is one of the key elements of the future energy supply of mankind. Researchers in the field of photovoltaics are faced with the problem how to increase the efficiency of this conversion process for reasonable cost. The solution is related to optics to a large part. By optimizing the optics, the number of photons which are converted by the semiconductor material can be maximized. First of all, optical approaches are necessary to avoid losses by reflection or unwanted absorption which does not generate charge carriers. As an example, part of the solar radiation incident on a typical PV module made out of crystalline silicon solar cells can already be reflected at the air-glass interface or be absorbed in the glass pane or the lamination material before reaching the solar cell. Further, the light has to be trapped efficiently within the cell. And the photon energy is far away from being optimized. Due to the large range of wavelengths in the solar spectrum almost all photons have the wrong energy because the photon energy may exceed the bandgap energy of the semiconductor or fall below that. In the first case, the photon generates an electron-hole pair and the excess energy is thermalized, in the second case the photon is transmitted or absorbed without generating an electron-hole pair, e.g. by free carriers. Only photons with exactly the energy of the band gap of the semiconductor are generating electron-hole pairs with the maximum efficiency. Thus, the researcher is faced with the need for spectral control of the incident photons.
This paper gives an overview on the various optical approaches in order to optimize the efficiency of PV cells. Only purely optical approaches in which the electronic semiconductor device structure is not affected shall be described here. Readers which are interested in multijunction solar cells, multiple exciton solar cells intermediate band solar cells, sometimes using nanotechnology etc. are referred to e.g. Here, the addressed topics are antireflection, light trapping, solar concentration, spectral management, and combinations thereof.

2 Properties of solar irradiation on earth and consequences for optical components The properties of the solar radiation which we receive on earth depend on the diameters of sun and earth, on the sun-earth distance, on the surface temperature of the sun, and on the interaction of the radiation with the earth's atmosphere. The solar spectral irradiance measured a top of the earth's atmosphere can be rather well approximated by a blackbody spectrum of a body with a temperature of $5762 \mathrm{~K}$ seen under a solid angle $\Omega_{\mathrm{s}}$ of $2.16 \times 10^{5}$

This extremely small solid angle is due to the large distance between sun and earth and implies that the direct solar irradiance is very well collimated.

The fact, that the extraterrestrial solar spectrum resembles blackbody radiation is reflected by the spectral distribution which is extremely broadband from an optical point of view, being extended over wavelengths from below $300 \mathrm{~nm}$ to more than $3000 \mathrm{~nm}$. The interaction of the solar 
radiation with the earth's atmosphere is defined by absorption and scattering by gases like $\mathrm{CO}_{2}, \mathrm{~N}_{2} \mathrm{O}, \mathrm{CH}_{4}$, fluorinated carbon dioxides, ozone, oxygen, and finally dust and aerosols. It is obvious that the path length of the solar radiation through the atmosphere is of major importance. Therefore, the solar reference spectra are defined for different air mass values which indicate the path length through the atmosphere, e.g. AM 1.5 is a spectrum obtained for solar radiation after being transmitted through 1.5 times the atmosphere thickness corresponding to a solar zenith angle of $48.19^{\circ}$. Reference spectra for PV performance evaluation are currently in ASTM G173-03 or in ISO $9845-1$ for the extraterrestrial spectrum, the terrestrial global solar irradiance on a surface tilted by $37^{\circ}$ toward the equator facing the sun, and for direct normal irradiance plus irradiance from circumsolar radiation (Fig. 1). They can be found at the web based renewable resource data center of NREL Of course, as the terrestrial spectra change with the properties of the atmosphere, the reference spectra refer also to reference conditions of the atmosphere. Spectral variations are inevitable and may influence the performance of PV systems.

The large spectral band width makes the task of an optical engineer difficult. For example, many of the thin film designs developed for applications in the visible cannot be used because they may have undesirable optical effects if the wavelength reaches or exceeds the double of the design wavelength. Another important aspect resulting from the large band width of the solar spectrum is the short optical coherence length in the order of magnitude of a micrometre. Therefore, in all optical simulations optical coherence considerations have to be taken into account. Calculations based on the complex degree of coherence are not possible in many cases or very time consuming at least. Therefore, very often some parts of the PV system are calculated assuming complete optical coherence, e.g. thin film systems, while in others the incoherent superposition of intensities is assumed, e.g. multiple reflections within a glass superstrate. Most critical is the case when the dimensions of op-

\section{ASTM G173-03 Reference Spectra}

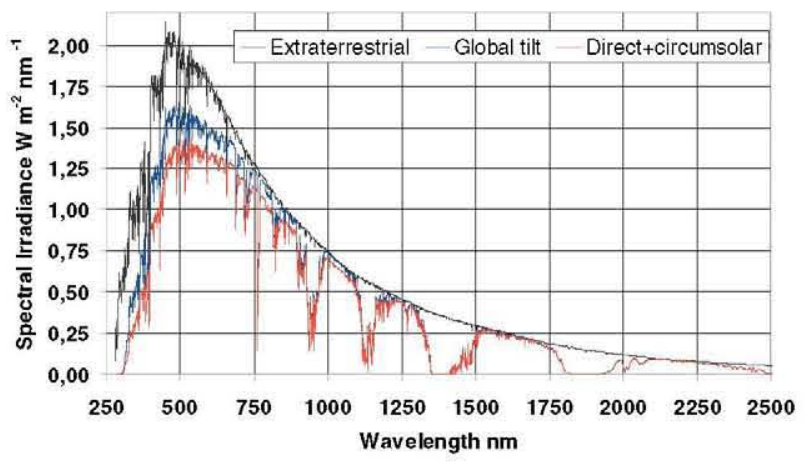

Figure 1 (online colour at: www.pss-a.com) Reference spectra for solar radiation, extraterrestrial, terrestrial global and direct tical structures are in the range of the coherence length, the already mentioned micrometre.

The terrestrial solar irradiance is in the range of $1 \mathrm{~kW} / \mathrm{m}^{2}$. This indicates that very large apertures for solar systems are needed if solar energy systems shall contribute significantly to the coverage of our energy demand. Therefore, any optical solution which is developed to improve the conversion efficiency of PV systems has to be producible on very large areas for low cost.

3 Antireflection At any interface between two media with different refractive indices part of the incident radiation is reflected. The degree of reflection scales with the difference of the refractive indices $\Delta n$ of the two adjacent media. Thus, in a PV module made out of crystalline PV cells there are two interfaces at which larger losses of a PV system due to reflection would occur without counter measures: the air-glass interface and the embedding material-solar cell interface. The embedding material is typically ethylene vinylene acetate (EVA) with a refractive index which matches very well that of glass. The reduction of reflection can be obtained by either interference coatings or surface-relief structures. In the latter case, the structure dimensions with respect to the design wavelengths play an important role. If they are in the range of a wavelength or larger, the incident radiation is scattered or diffracted or refracted by the surface-relief structure and thus changes the direction of propagation. If the dimensions are subwavelength, an effective medium is formed which has an optical effect similar to interference coatings. For the air-glass interface in PV systems, all the approaches: interference layers, subwavelength structures and structures in the millimetre range have been proposed If the spectral width for which an air-glass interface has to be made antireflective exceeds one octave, only refractive indices of the interference layers between those of the two materials forming the interface can be used. Therefore, it was proposed to use porous layers in order to achieve the low effective refractive indices required for an air-glass interface (Fig. 2). Of course, the best would be to have a graded index between air and glass but the most suitable

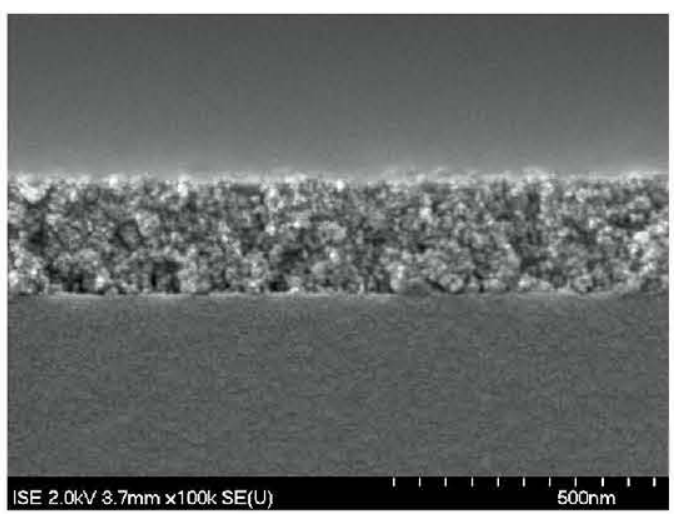

Figure 2 SEM picture of a cross section of a porous single layer antireflective coating. 


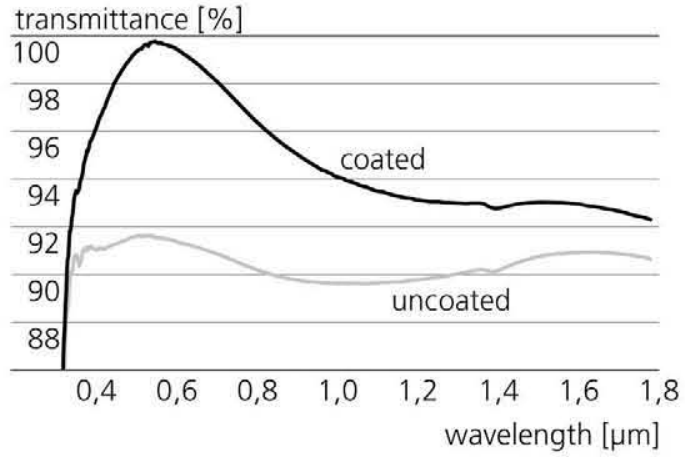

Figure 3 Transmission spectra of low-iron glass panes with and without a porous antireflective coating on both surfaces.

solution with respect to cost and mechanical performance is a quarter wave single layer with a refractive index of 1.25-1.30. With such an interference coating, a significant reduction of the reflection is obtained over a large spectral range as required in most $\mathrm{PV}$ applications although antireflection in its true sense $(R=0)$ can theoretically only be achieved for the design wavelength (Fig. 3). This approach is now used in various commercially available AR coatings on glass for solar applications

Subwavelength structures could be originated and replicated in polymer materials on large areas and with good optical properties The replication of such small structures in durable materials like glass is aspired but was not possible areas larger than a few square millimetres so far. Surface-relief structures in the millimetre range make use of three optical effects. Firstly, radiation which is incident at rather large angles of incidence with respect to the normal of the glass pane has relatively small local angles of incidence and thus a smaller reflectance (Fig. 4a). Secondly, radiation which is reflected at the first interaction with the glass surface gets a second chance to be transmitted light for certain angles of incidence (Fig. 4b). Thirdly, reflected light from the solar cells, e.g. from the bus bars, may be totally internally reflected to the solar cell (Fig. 4c). In total this leads to an increase of the annual output of the PV system. It is important to note this type of antireflection system is beneficial only if the absorber (the solar cell) is optically matched to the glass pane.

The task of antireflection at the interface between EVA and the crystalline silicon solar cell is very well fulfilled by a quarter-wave single layer $\mathrm{SiN}: \mathrm{H}$ coating together with an etched light trapping structure (see Section 4). There are the following advantages of the $\mathrm{SiN}: \mathrm{H}$ coating: it can be tuned in its refractive index and it is a very good passivation layer. Therefore, this material outperformed other high refractive index materials which were used earlier like $\mathrm{TiO}_{2}$ and $\mathrm{Ta}_{2} \mathrm{O}_{5}$. In the case of mono crystalline silicon wafers which show very well suited random pyramids after etching very low reflectance $<3 \%$ over the spectral range of $300-1100 \mathrm{~nm}$ can be obtained. In an experimental study, it was also proposed to use periodic subwavelength
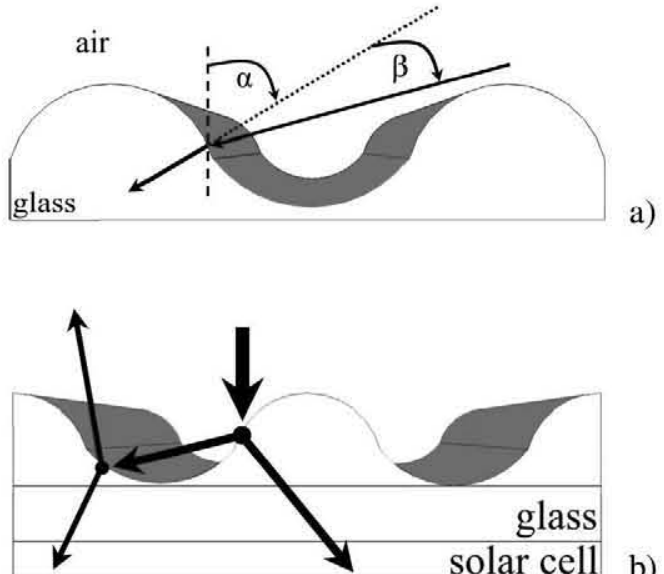

b)

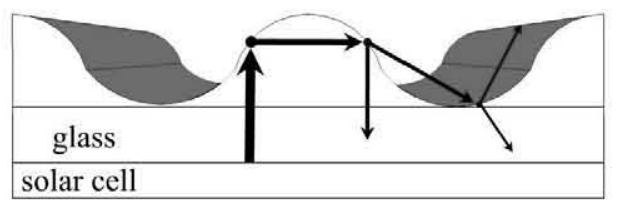

c)

Figure 4 Illustration of the optical effects of a glass surface which has surface-relief structures in the millimetre range.

structures for making silicon antireflective Again, it is questionable whether this approach can be competitive with respect to cost and large-scale production.

In inorganic thin film solar cells, the situation is different insofar as the functional layers are coated on a substrate or superstrate directly and transparent electrode layers (TCO-transparent conductive oxide) are used. For example, the device structure of an a-Si pin solar cell is sketched in Fig. 5. Antireflection of and light trapping in the solar cell material is made by the roughness of the TCO layer which is intentionally produced during film growth or via etching. A very detailed analysis of the near-field effects in a-Si cell structured with randomly textured interfaces was published recently As an example the surface of a widely used TCO film (SnO:F) is shown in Fig. 6.

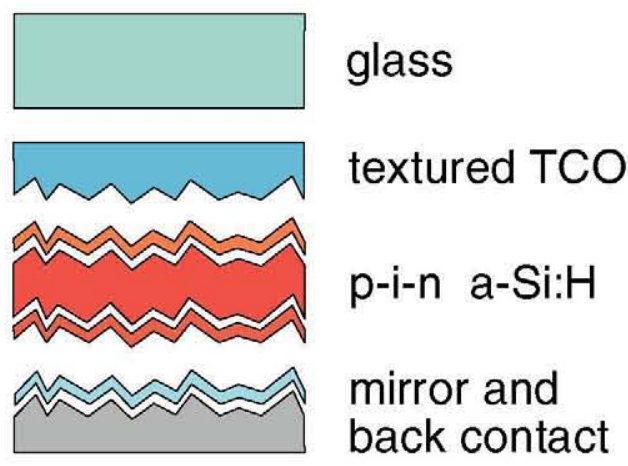

Figure $\mathbf{5}$ (online colour at: www.pss-a.com) Sketch of an a-Si pin thin film solar cell. 


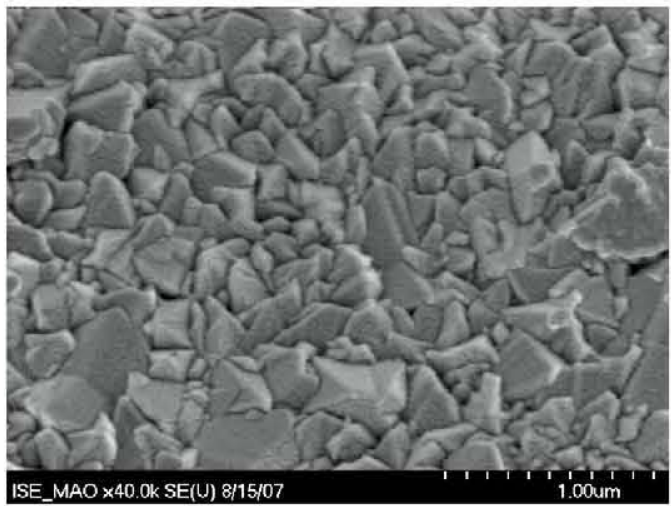

Figure 6 SEM picture of the surface of a TCO coating for thin film solar cells.

The optimum structure is still subject of research

Correct simulation results can only be expected when the optical properties are simulated rigorously: a difficult task for aperiodic structures. As soon as the thin film become even thinner, e.g. in the case of organic solar cells, the whole film system has to be optimised as a stack of interference layers in order to reduce reflection losses and to increase absorptance in the active layer.

4 Light trapping In crystalline silicon, an indirectgap semiconductor, light is not absorbed very well. Thus, in the mid 1970's the benefits of increased optical path length at constant or even reduced cell thickness for $\mathrm{c}-\mathrm{Si}$ solar cells were discussed and shortly later surface textured PV cells for light trapping were already commercialized Arndt et al. developed a surface texture which had a very low reflectance after being coated with $\mathrm{Ta}_{2} \mathrm{O}_{5}$ for wavelengths from $500 \mathrm{~nm}$ to $1 \mu \mathrm{m}$ and showed that the increased the optical path length results in an increased charge carrier generation rate. Yablonovitch and Cody adopted a statistical mechanical approach towards the optics of textured (assumed Lambertian, i.e. perfectly diffusive) and inhomogeneous optical sheets and calculated the intensity inside the textured sheet which is immersed in black body radiation The intensity inside the sheet is enhanced by a factor of $n^{2}(x)$ with respect to the intensity in the surrounding air. $n(x)$ is the local refractive index. For lowly absorbed photons and provided the solar cells have a perfect light reflector in the back side the effective path length can be increased by a factor as $4 n^{2}$, one factor 2 coming form the average inclination of the rays due to the texture, a second factor two coming form the reflection in the back mirror and a factor $n^{2}$ comes form the total internal reflection most of the rays experience at the from surface when they hit there above the limit angle This is a remarkable value (about 46) taking the high refractive index of silicon into account. The value becomes only $2 n^{2}$ if no perfect mirror is in the back face but has a Lambertian texture like the one at the front face. Therefore, periodic surface-relief structures were manufactured for test cells using photolithography and random structures

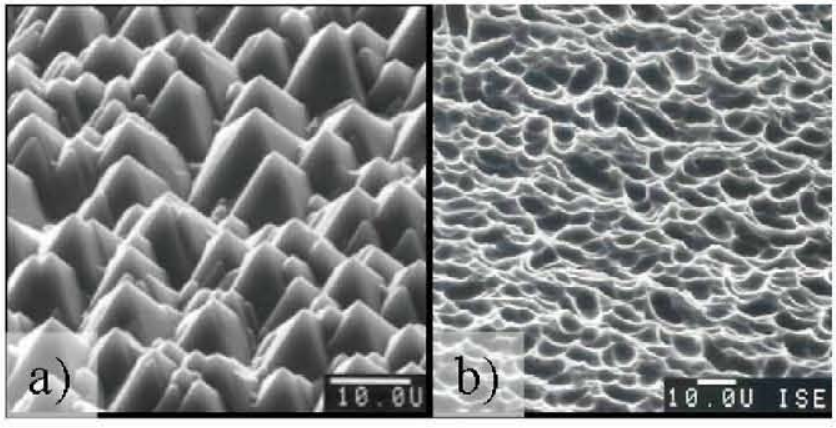

Figure 7 a) SEM picture of the texture etched on mono crystalline silicon solar cells; b) SEM picture of the texture etched on multi crystalline silicon solar cells.

are made in large scale solar cell production. Mono crystalline silicon can be anisotropically etched in alkaline solutions forming random pyramids with very good optical properties (Fig. 7a). Multi crystalline silicon is etched in acidic solutions because an anisotropic etching process would only be good for the silicon grains with the right orientation towards the wafer surface. The structure profile resulting from the random acidic etch process reduces the reflection significantly but leaves room for improvement because of the large quasi planar parts of the surface (Fig. 7b). Using a plasma etch process in combination with a photolithographically defined etch resist pattern results in a better performance but is not industrially viable. In inorganic thin film solar cells, light trapping is done by the rough TCO surface. For a better understanding, work is also done on periodic light trapping structures This closes the circle to earlier approaches to use diffraction gratings for light trapping The idea is to diffract incident radiation which can be converted but is hardly absorbed into the first or second diffraction order propagating almost parallel to the cell surface. One advantage of the diffraction grating with respect to the random textures is that it can be very shallow thus allowing for very thin active layers of the solar cell. Morf and Heine have shown that symmetric grating structure profiles are not the most efficient gratings because they present strong the diffraction in the zero order after reflection in the upper face Thus, the symmetry has to be broken, e.g. by using a blazed grating (Fig. 8). It is worth noticing that more recently diffraction gratings were taken again into consideration, e.g. as light trapping structures for organic solar cells or for thin film a-Si:H solar cells Nevertheless, diffractive gratings are strongly wavelength and direction dependent and can hardly compete with random textures both theoretically and in practice.

5 Solar concentration Concentration of solar radiation for PV applications was always motivated by the potential to reduce cost and to increase efficiency

The cost reduction is thought due to the replacement of expensive PV cell material by lower cost optical systems covering the receiver aperture. This argument is espe- 

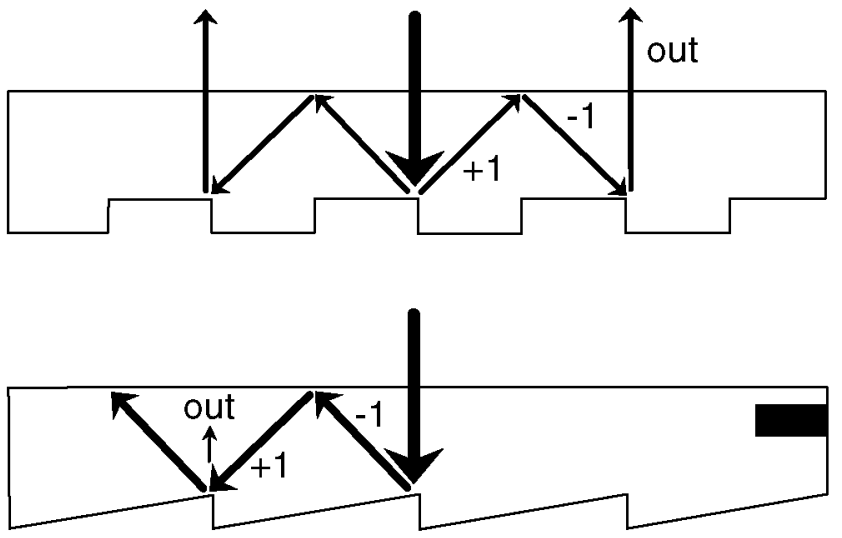

Figure 8 (online colour at: www.pss-a.com) Sketch indicating the advantage of a blazed grating in comparison to a symmetrical grating profile (here rectangular) for light trapping in solar cells.

cially true since very highly efficient but also expensive multijunction III-V concentrator solar cells became available The increased efficiency is related to the increase of the open-circuit voltage of the solar cell with concentration. If we assume a linear dependence of the photocurrent $I_{\mathrm{L}}$ on the concentration $C$, i.e., and a simple one-exponential model to describe the solar cell, we find:

$$
V_{\mathrm{oc}, C}=V_{\mathrm{oc}, 1}+m \frac{k T}{e} \ln C
$$

with $V_{\text {oc }, C}$ and $V_{\text {oc, }, 1}$ being the open-circuit voltages at concentrations $C$ and 1 respectively, and $\mathrm{m}$ being the diode's ideality factor.

Equation (1) can only be used to understand the general dependence. In reality, differences from the logarithmic increase in $V_{o c}$ with concentration are found, e.g. due to some non linear dependence of recombination processes on the carrier concentration and to ohmic drops inside the cell (even at open circuit).

A very good comprehensive introduction to the optics for photovoltaic concentrator systems can be found in the chapter "Concentrator optics for the next generation photovoltaics" from Benitez and Miñano and a more detailed one Therefore, only the basic principles shall be described here using Fig. 9 for illustration and following the description given We assume a concentrator pointing in the direction given by the unit vector $\boldsymbol{v}$. Thus, $\boldsymbol{v}$ is the normal incidence direction vector. The concentrator shall concentrate all radiation which is incident on its entry aperture $A_{\text {in }}$ with angles of incidence $\leq \theta_{\text {in }}$ (the so-called acceptance angle) onto the exit area $A_{\text {out }}$ with angles of incidence $\leq \theta_{\text {out }}$ at the exit area. Both areas are assumed to be the projection on planes normal to $\boldsymbol{v}$. In concentrator photovoltaics (CPV), the exit area of the concentrator normally represents the PV cell area. The geometrical concentration is defined as $C_{\mathrm{g}}=A_{\text {in }} / A_{\text {out }}$. From the theorem of the conservation of the étendue (or also called Liouville's theorem) which holds for all non-scattering loss-free passive concentrators inequality

$$
C_{\mathrm{g}} \sin ^{2} \theta_{\text {in }} \leq n^{2} \sin ^{2} \theta_{\text {out }}
$$

with $n$ being the refractive index of the concentrator $n \geq 1$ and the medium for which $\theta_{\mathrm{in}}$ is defined having a refractive index of 1 (e.g. air). The equality in (2) is the thermodynamic concentration limit for a given acceptance angle. It is the sine condition in the geometrical theory of optical imaging which was first derived by Clausius from thermodynamical considerations but is also known Abbe's sine condition. How $\theta_{\mathrm{in}}, \theta_{\text {out }}$ and $C_{\mathrm{g}}$ are chosen in practise depends on many factors including the definition of $C_{\mathrm{g}}$, of the effective acceptance angle, and also of the optimum PV concentrator performance.

With the maximum for $\theta_{\text {out }}=90^{\circ}$, the case of isotropic illumination of the exit area and the angle under which we would see on earth the sun disc without perturbation through the atmosphere $\alpha_{\mathrm{S}} \cong 0.27^{\circ}=\theta_{\text {in }}$ we can calculate the maximum concentration:

$$
C_{\mathrm{g}, \max }=n^{2} \cdot 46200 .
$$

The basis of the sine condition is the continuity of the energy flux density per solid angle in regions with the same refractive index. Thus it is equivalent to calculate $\mathrm{C}_{\mathrm{g} \text { max }}$ by taking the solid angles $\Omega_{\mathrm{S}}$ (sun on entry aperture, see Section 2) and $\pi$ (isotropic illumination of exit area) into account The maximum concentration achievable on Earth is very high. Actually 56,000 times the solar irradiance has been achieved in an experiment but in practical cases the obtained values are much lower. Most of the high concentration CPV modules which are commercialized or close to commercialisation have a concentration of $400-1200$.
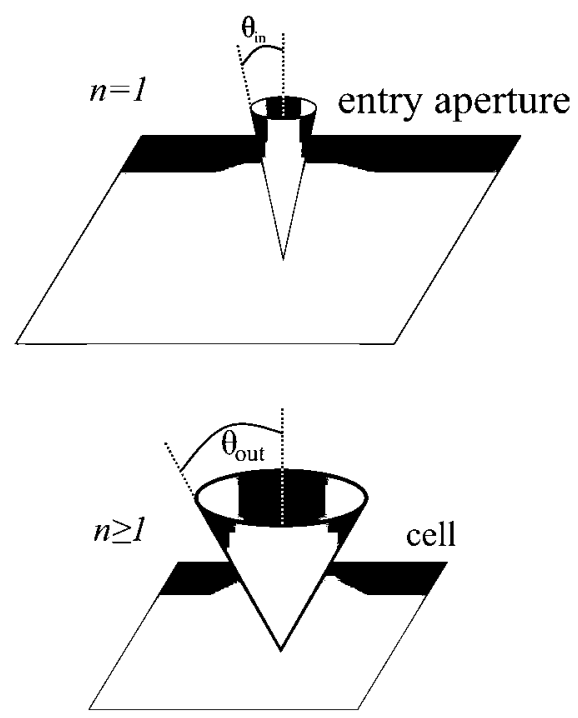

Figure 9 Illustration of the concentrator geometry. 
optical designs had to be developed for photovoltaic applications. One of the reasons for that is the large spectral bandwidth of the solar radiation. Another, that only very low cost solutions are commercially viable. Optical thin film systems for antireflection and high reflection, random micro structures for light trapping and engineered surfacerelief structures with dimensions in the order of one millimetre for solar concentrators are examples of optical components which were successfully introduced into the market yet. Demanding nano and micro structures, for example diffraction gratings, were used scientifically but not on a broad commercial basis so far. Nevertheless, with a better scientific understanding, better materials and more advanced production methods also more sophisticated photonic elements for increasing the efficiency of photovoltaic systems will be worth. The interest in photonics for photovoltaics is currently experiencing a comeback.

Acknowledgements The authors acknowledge the very fruitful discussions with S. Glunz, Fraunhofer ISE, concerning light trapping in silicon solar cells. A.G. further acknowledges the partial financial support of this work by the Deutsche Forschungsgemeinschaft (PAK88) and the Federal Ministry of Education and Research (Nanovolt) A.L. acknowledges the support of the CSD2006-0004 CONSOLIDER National Spanish grant and the S-05050/ENE/0310 grant of the Comunidad de Madrid.

\section{References}

A. Marti and A. Luque (ed.), Next Generation Photovoltaics (Institute of Physics Publishing, Bristol, 2004).

A. d. Vos, Endoreversible Thermodynamics of Solar Energy Conversion (Oxford University Press, New York, 1992). http://rredc.nrel.gov/solar/spectra/am1.5/

C. Heine and R. H. Morf, Appl. Opt. 34, 2476 (1995).

A. Gombert, W. Glaubitt, K. Rose, J. Dreibholz, C. Zanke, B. Bläsi, A. Heinzel, W. Horbelt, D. Sporn, W. Döll, V. Wittwer, and J. Luther, Solar Energy 62, 177 (1998).

U. Blieske, T. Doege, M. Neander, A. Prat, and P. Gayout, "Textured plate with high light transmission", Patent WO 03/046617 A1 (2001).

www.centrosolarglas.de

www.sunarc.net

www.interpane.net

A. Gombert, B. Bläsi, Ch. Bühler, P. Nitz, J. Mick, W. Hoßfeld, and M. Niggemann, Opt. Eng. 43, 2525 (2004).

P. Lalanne and G. M. Morris, Nanotechnology 8, 53 (1997).

K. Bittkau, T. Beckers, S. Fahr, C. Rockstuhl, F. L. Lederer, and R. Carius, phys. stat. sol. (a) 205(12), 2766 (2008), this issue.

C. Haase and H. Stiebig, Proc. SPIE 6197, 619705 (2006).

D. Redfield, Appl. Phys. Lett. 25, 647 (1974).

R. A. Arndt, J. F. Allison, J. G. Haynos, and A. Meulenberg, in: Proc. 11th IEEE Photovoltaics Specialists Conf., 40 (1975).

E. Yablonovitch and G. D. Cody, IEEE Trans. Electron Devices 29, 300 (1982).

A. Luque, Sol. Energy Mater. 23, 152 (1991).

P. Campbell and M. A. Green, J. Appl. Phys. 62, 243 (1987).
A. Hauser, I. Melnyk, P. Fath, S. Narayanan, S. Roberts, and T. M. Bruton, in: Proc. 3th World Conference on Photovoltaic Energy Conversion, Osaka, Japan, 2003, pp. $1447-$ 1450 .

O. Schultz, G. Emanuel, S. W. Glunz, and G. P. Willeke, in: Proc. 3th World Conference on Photovoltaic Energy Conversion, Osaka, Japan, 2003, pp. 1360-1363.

O. Schultz, S. W. Glunz, and G. Willeke, Prog. Photovolt. 12, 553 (2004)

P. Sheng, A. N. Bloch, and R. S. Stepleman, Appl. Phys. Lett. 43, 579 (1983).

M. Niggemann, M. Glatthaar, A. Gombert, A. Hinsch, and V. Wittwer, Thin Solid Films 451/452, 619 (2004).

M. Kroll, S. Fahr, C. Helgert, C. Rockstuhl, F. Lederer, and Thomas Pertsch, phys. stat. sol. (a) 205(12), 2777 (2008), this issue.

I. Tobías, A. Luque, and A. Martí, J. Appl. Phys. 104, 034502 (2008)

M. Yamaguchi and A. Luque, IEEE Trans. Electron Devices 46, 2139 (1999).

N. H Karam, R. A. Sherif, and R. R. King, in: Concentrator Photovoltaics, edited by A. Luque and V. Andreev, Springer Series in Optical Sciences, Vol. 130 (Springer-Verlag, Berlin, 2007), chap. 10.

A. Luque, Solar Cells and Optics for Photovoltaic Concentration (Adam Hilger, Bristol, 1989).

T. Jannson and R. Winston, J. Opt. Soc. Am. A 3, 7 (1986).

M. Born and E. Wolf, Principles of Optics (Pergamon Press, Oxford, 1984), p. 167

P. Würfel, Physics of Solar Cells (Wiley-VCH Verlag, Weinheim, 2005).

P. Gleckman, J. Ogallagher, and R. Winston, Nature 339, 198 (1989)

R. Leutz and A. Suzuki, Nonimaging Fresnel lenses - Design and Performance of Solar Concentrators, Springer Series in Optical Sciences, Vol. 83 (Springer-Verlag, Berlin, 2001).

J. C. Goldschmidt, M. Peters, L. Prönneke, L. Steidl, R. Zentel, A. Gombert, S. Glunz, G. Willeke, and U. Rau, phys. stat. sol. (a) 205(12), 2811 (2008), this issue.

L. W. James, Use of imaging refractive secondaries in photovoltaic concentrators, SAND89-7029, Albuquerque, NM, 1989.

J. M. Gordon and D. Feuermann, Appl. Opt. 44, 2327 (2005).

J. M. Gordon, in: Concentrator Photovoltaics, edited by A. Luque and V. Andreev, Springer Series in Optical Sciences, Vol. 130 (Springer-Verlag, Berlin, 2007), chap. 6.

J. C. Miñano, J. C. González, and P. Benítez, Appl. Opt. 34, $7850(1995)$

A. W. Bett, B. Burger, F. Dimroth, G. Siefer, and H. Lerchenmüller, in: Proc. 4th World Conference on Photovoltaic Energy Conversion, Waikoloa, Hawaii, 2006, pp. $745-$ 748

M. Yamaguchi, K. Nishimura, T. Sasaki, H. Suzuki, K. Arafune, N. Kojima, Y. Shsita, Y. Okada, A. Yamamoto, T. Takamoto, and K. Araki, Solar Energy 82, 173 (2008).

F. Rubio, M. Martínez, R. Coronado, J. L. Pachón, and P. Banda, Deploying CPV power plants: The ISFOC experiences, in: 33rd PVSC, San Diego, 2008, in press. 
In principle, for photovoltaic spectrum splitting the question is how to arrange filtering elements and cells. Luckily, PV cells can also serve as filtering elements. A straightforward arrangement is to cascade planar cells as it is done in tandem or multijunction cells (Fig. 11a). For a lateral arrangement (Fig. 11b), an extra filtering element is needed because the PV cells cannot act as filters. Often, for this extra filter diffractive or holographic elements were proposed [e.g. 46-51]. Newer approaches include photonic crystals as dispersive elements In non-coplanar arrangements as proposed by Elliot (Fig. 11c) or by Ortabasi [54], where the cells cover the inner surface of an integrating hollow sphere, again the spectral filtering is done by the solar cells of different bandgap. A fourth possibility is the luminescent filtering and concentration where solar cells are attached in various configurations at the edges or the bottom of a light guide with fluorescent dye molecules (Fig. 11d) A good review on fluorescent concentrators has been given by Zastrow An outstanding property of luminescent concentrators is the suitability to concentrate diffuse radiation. The interest in lu-

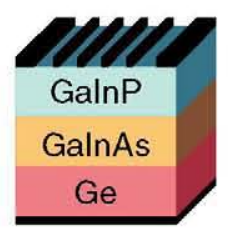

a)

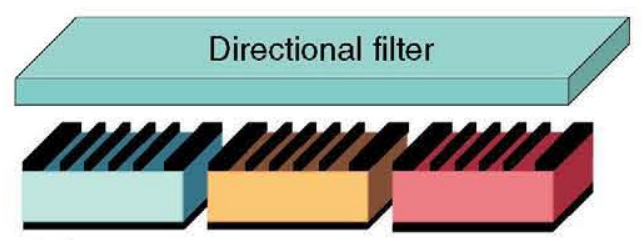

b)
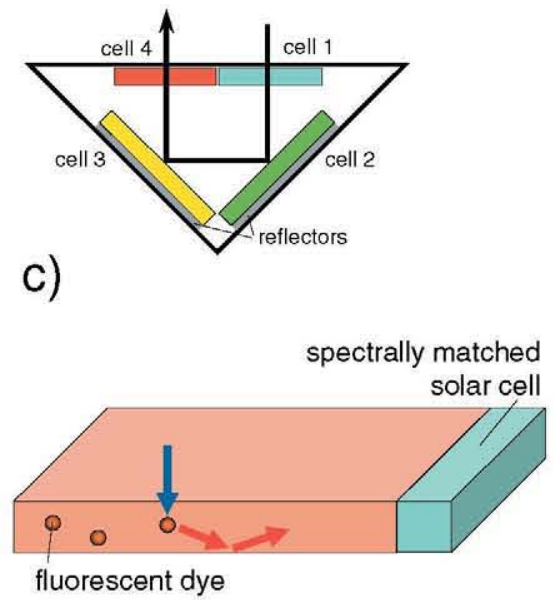

d)

Figure 11 (online colour at: www.pss-a.com) Arrangements of PV cells for spectrum splitting. minescent concentrators has grown recently, more than 20 years after the first phase of intense research, because of new ideas how to avoid losses. More details can be found in the mentioned publication related to this one

Numerous publications exist on diffraction gratings or holographic optical elements for spectral management and concentration in PV systems. A comprehensive overview is given by Riccobono and Ludman The basic idea is to use a grating as dispersive element to separate the spectral components of the solar radiation laterally and to combine it with the possibility to concentrate radiation with a small spectral bandwidth by using a hologram. Volume phase holograms are films in which a permanent pattern is recorded as a variation of the refractive index. Due to the fact, that the film thickness exceeds the recording and reconstruction wavelengths by orders of magnitude, they show a pronounced Bragg effect making them suitable for the task of spectrum splitting for PV systems: firstly, their diffraction efficiency can reach values close to $100 \%$, secondly, they can be multiplexed, or in other words multiple holograms in one film are possible with relatively low cross-talk between the holograms. A typical arrangement for a transmission hologram is shown in Fig. 12. From this sketch, one can imagine already that high concentration systems are not very compact. Nevertheless, one advantage of a set-up as shown in Fig. 12 is the possibility to filter out non-convertible infrared radiation keeping the solar cells at a lower temperature in operation. A more compact design was proposed by Fröhlich in which a stack of two holographic concentrator lens plates with $7 \times 7$ lenses each focused a short and a long wavelength band of the solar radiation on the respective PV cells at a geometric concentration of 49 . A very compact low concentrating holographic PV module is commercially available

7 Summary Photonics plays an important role for the optimization of photovoltaic systems. In most cases new

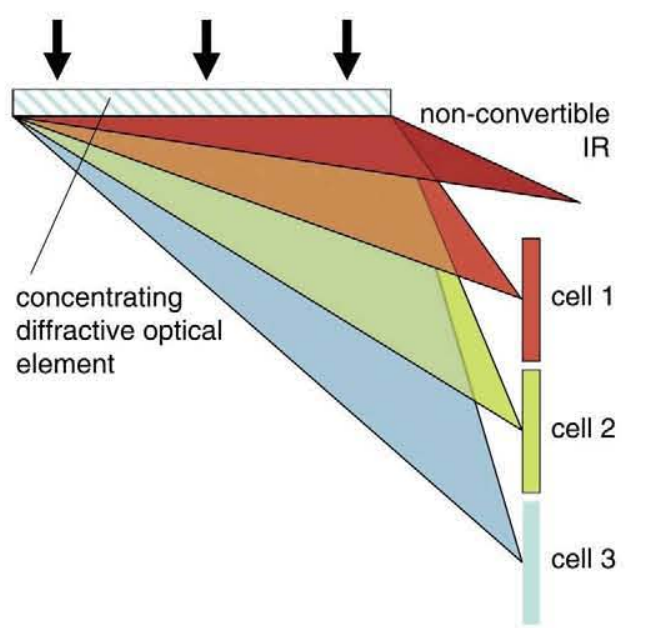

Figure 12 (online colour at: www.pss-a.com) Sketch of a holographic spectral filter/concentrator. 
There are many approaches for solar concentrators depending on the optical principles lying behind and on the two principal classes, imaging and nonimaging. This classification is made by Leutz and Suzuki and gives a very good overview. The optical principles are reflection, refraction, dispersion/diffraction, and fluorescence. Diffractive concentrators are described in the next section, fluorescence concentrators in a publication related to this one Therefore, we will concentrate here on refractive and reflective concentrators and combinations thereof. The classical types representing each of the principles are the Fresnel lens and the parabolic mirror. These two cannot fulfil perfectly all the requirements on solar concentrators for PV, e.g. high optical efficiency and concentration, uniform illumination, suitability for low-cost mass production, insensitivity to tracking and manufacturing errors and to atmospheric variations of the incident direct solar radiation, suitability to be arranged in modules, durability, low weight, and low aspect ratio (height/width) in order to be able to produce flat modules. Therefore, more sophisticated designs have been developed. A first step to improve the illumination uniformity and the acceptance angle was the introduction of a secondary concentrator Further steps include the combination of primary and secondary concentrators in one system. In the past non-imaging designs were regarded as being superior as they can be ideal concentrators at the thermodynamic limit while imaging concentrators are generally not ideal Recently, it was shown that with an aplanatic imaging design very high flux level, as good or even exceeding those of non-imaging practical designs, could be obtained A superb example for a nonimaging concentrator is the RXI concentrator designed by Miñano The RXI concentrator uses refraction, reflection, and total internal reflection (the same surface as the refractive one) and has a very low aspect ratio at high concentration. The aplanatic imaging design of Gordon and Feuerbach resulted in a compactness similar to the RXI concentrator and in high flux. The research for optical concentrators which are the best compromise to fulfil the above mentioned requirements is still going on.

Currently, there are many research institutes and companies around the world working on the market introduction of CPV. The experimental set-ups of the last 30 years are now replaced by various commercial products as shown e.g. in Fig. 10. High peak efficiencies of $27-29 \%$ at a concentration of approximately 500 have already been measured for modules with flat or dome shaped Fresnel lenses as primary concentrator and III-V multijunction solar cells An important step forward in this field is the establishment of the new ISFOC institute in Puertollano (Spain) that has issued international calls for tenders to install $3 \mathrm{MW}$ of concentrators with the aim of helping companies to set up pilot lines for concentrator manufacturing. Seven companies have been awarded with contacts and in the present days the installation of the first $0.8 \mathrm{MW}$ is under way (see Fig. 10). First measurements of concentrator arrays have been performed in Puertollano
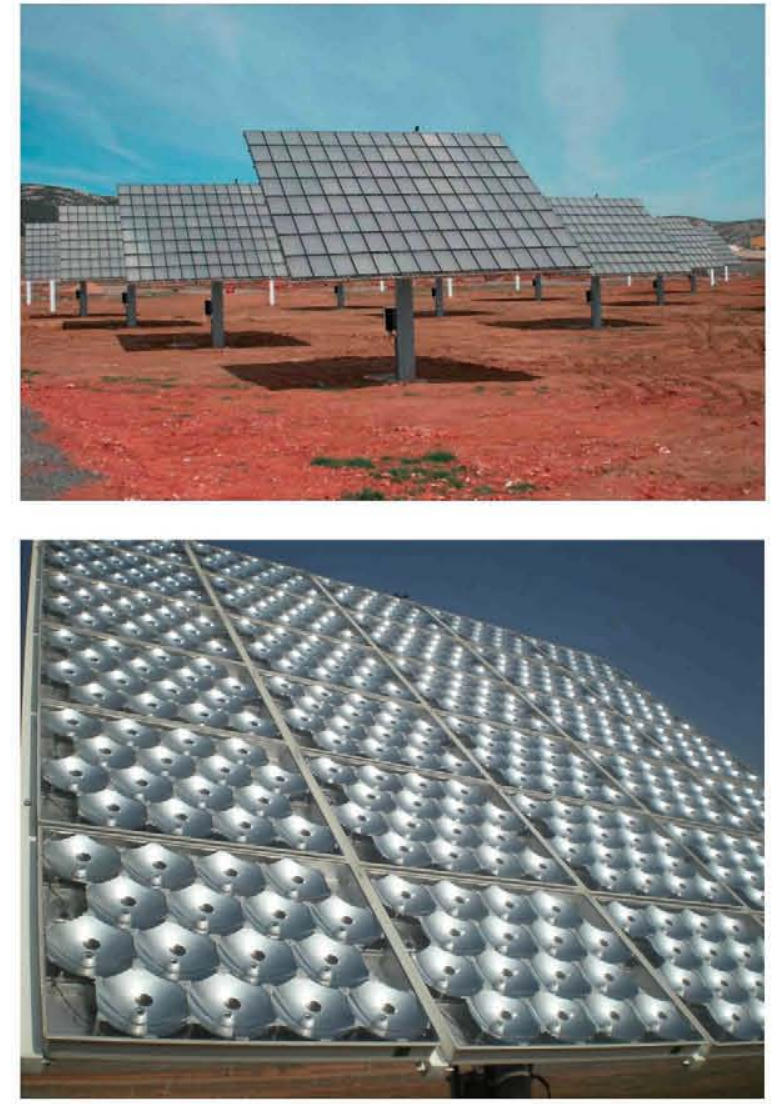

Figure 10 (online colour at: www.pss-a.com) Top: Concentrator photovoltaic (CPV) systems of the company Concentrix Solar GmbH installed at the ISFOC. Bottom: CPV modules of the company Solfocus Inc all installed at the ISFOC (photo courtesy: ISFOC)

Due to the sensitivity of CPV modules to site, seasonal and atmospheric variations the peak efficiency alone is not the right measurement for the performance of a CPV system. Therefore, the right rating method for CPV systems is still discussed.

6 Spectral management A single bandgap solar cell cannot convert the full solar spectrum with a high efficiency. High efficiencies require radiation of a very small spectral interval, ideally monochromatic, and, as shown, concentration. Thus, spectral management, often in combination with concentration, is very important. A very good review paper on this topic was published by Imenes and Mills Their paper has a strong focus on spectral management between photovoltaic and photothermal conversion but also a PV/PV spectrum splitting section. Within this paper, mainly optical approaches of spectral management between solar cells or subcells with different bandgap, and thermophotovoltaics shall be described. Generally, the better utilization of the full solar spectrum is described in

The important topic of up and down conversion was first published in and is described also in two publications related to this one 
A. G. Imenes and D. R. Mills, Sol. Energy Mater. Sol. Cells 84, 19 (2004).

T. Trupke, M. A. Green, and P. Würfel, J. Appl. Phys. 92, 1668 (2002).

T. Trupke, M. A. Green, and P. Würfel, J. Appl. Phys. 92, 4117 (2002)

B. Ahrens, P. Löper, J. C. Goldschmidt, S. Glunz, B. Henke, P. T. Miclea, and S. Schweizer, phys. stat. sol. (a) 205(12), 2822 (2008), this issue.

J. Ludman, Appl. Opt. 21, 3057 (1982).

W. H. Bloss, M. Griesinger, and E. R. Reinhardt, Appl. Opt. 21, 3729 (1982).

T. Jannson and J. Jannson, in: Proc. SPIE 523, 219-226 (1985).

C. G. Stojanoff, R. Kubitzek, S. Tropartz, K. Fröhlich, and O. Brasseur, Proc. SPIE 1536, 206-214 (1991).

K. Fröhlich, U. Wagemann, J. Schulat, H. Schütte, and C. G. Stojanoff, Proc. SPIE 2255, 812-821 (1995).
J. E. Ludman, J. R. Riccobono, I. V. Semenova, N. O. Reinhand, W. Tai, X. L. Li, G. Syphers, E. Rallis, G. Sliker, and J. G. Martin, Solar Energy 60, 1 (1997).

A. Bielawny, P. T. Miclea, A. v. Rhein, R. B. Wehrspohn, S. v. Riesen, and S. Glunz, Proc. SPIE 6197, 619704 (2006). M. E. Elliot, High efficiency photovoltaic assembly, Patent US 8,701,512 (1987).

U. Ortabasi, A. Lewandowski, R. McConnell, D. J. Aiken, P. L. Sharps, and B. G. Bovard, in: Proc. 29th IEEE Photovoltaic Specialists Conference, New Orleans, LA, 2002.

A. Goetzberger and W. Greubel, Appl. Phys. 16, 399 (1978).

A. Zastrow, Proc. SPIE 2255, 534-547 (1995)

J. R Riccobono and J. E. Ludman, in: Holography for the new millennium, edited by J. E. Ludman, H. J. Caulfield, and J. R. Riccobono (Springer, New York, 2002), pp. 157178.

www.prismsolar.com 